\author{
St ud i P hilos o p hi c a \\ Wr a t i s l a vi e n s i a \\ vol. XV, fasc. $1(2020)$ \\ https://doi.org/10.19195/1895-8001.15.1.1
}

OSENI TAIWO AFISI

ORCID: 0000-0001-7192-3134

Lagos State University

\title{
Re-echoing the Conservatism in Karl Popper's Piecemeal Engineering
}

\begin{abstract}
While Karl Popper highly valued the ability to invent a bold new form of theoretical thought, he warned us at the same time of the need to be cautious in action. Ambitions that are utopian or revolutionary seemed to Popper always unacceptable. We must always be open to reforming our practices, but we must attempt this slowly and piecemeal. Every change that we make we must hold open to criticism. However, change must be conservative and anti-revolutionary. This is the demand that while any one element of current culture may be criticised and rejected, it is important to do this work piecemeal and carefully. Only then does the audacity or boldness of rejecting a seemingly settled view amount to courage: for it reflects also significant conservation of values. Popper regarded no social change or scientific breakthrough as revolutionary. Even the process of conjecture and refutation, of trial and error, is a piecemeal method. Popper's piecemeal method depicts Otto Neurath's metaphor of not dismantling the whole ship except perhaps one plank at a time, replacing each plank as the effort goes on. Every step is as conservative as it is also bold, and that alone helps make it courageous. It is only through this conservatism, the piecemeal anti-revolutionary change, that people can become bold in how they experiment and replace some formerly received norm or idea.
\end{abstract}

Keywords: conservatism, piecemeal engineering, Popper, utopianism, change

\section{Introduction}

Within the development of his thinking, the issue of boldness came for Karl Popper together with courage, as can be seen in his audacity to have rejected the 
seemingly established philosophies of Plato, G.W.F. Hegel and Karl Marx. However, of significant value in Popper's views on how some formerly received norm or idea can be replaced, is a conservatism that enjoins one to be, on the one hand, critical, and yet, on the other, anti-revolutionary. Popper proposed that we should be bold in both conjecture and criticism, but piecemeal in method.

Popper esteemed both open criticism of any idea and boldness of theoretical thought. As a thinker, Popper was himself audacious enough to denounce the highly revered Republic of Plato to attack the ostensibly "scientific" interpretation of history of Karl Marx, terming it unscientific, to question the scientific status of the theory of psychoanalysis of Sigmund Freud and of the "individual psychology" of Alfred Adler, to upset the verification principle of the Vienna Circle and then to stand intellectually opposed to logical positivism. Popper himself boldly advanced new ways of thinking about the nature of science, about cosmology and whether the world is deterministic, about what probability is, about the meaning of quantum mechanics, and about the qualities of freedom and security in an intellectually open society. It is well known that criticism is the touchstone concept of Popper's philosophy. Every element of human thinking should be open to potential criticism, Popper believed. Likewise, Popper believed that every element of human practice should be open to potential criticism. Yet, however much Popper appreciated the ability to despatch a formerly received way of thinking or to invent a bold new form of theoretical thought, he at the same time professed the need to be cautious in action. Ambitions that are utopian or revolutionary seemed to Popper always unacceptable. We must always be open to reforming our practices, but we must attempt this slowly and piecemeal. Every change that we make we must hold open to criticism. It should always remain possible for us to judge that some past reform of ours was a misstep.

The balance between boldness and caution in Popper is complex. Caricatures of Popper's philosophy either entirely miss the balance or otherwise do not do justice to it. The balance must be grasped, however, in order to fairly assess what Popper's philosophy is. To grasp the balance is essential if we are to register how Popper's prescription for boldness actually involves a kind of courage.

The concept of courage is more demanding than that of boldness because courage relates to settled conviction, to deeply held values. A fearful or insecure person is not apt to act in ways that reflect settled conviction or deeply held values. Insecurity will trap a person into inaction or into obsequious followership. Fear is the emotion that prepares individuals to quickly alter not merely the mode of their action (if they do attempt it) but even the very principles upon which they would normally base what they do and are willing to do. By contrast, courageous persons, in some context in which courage is called for, while they may quickly alter what they do, will not alter the principles upon which they base what they are willing to do (Catton 2010: 5). They act courageously by acting in the face of evident adversity, and their action stems from deeply held values. Courage is not possible except that reflection is motivated by a desire to preserve some values. By contrast, the concept of boldness has to do with striking out in an unexpected direction. A bold person simply oversteps some previously settled way of acting (Catton 2010: 6). 
Popper's philosophy seems to offer an answer to the issue of how boldness is related to courage. In order to spell it out, however, one has to go beyond his basic rhetoric of conjectures and refutations. One has to focus on the conservative dispositions, the anti-revolutionary attitudes, the demand that while any one element of current culture may be criticised and rejected, it is important to do this work piecemeal and carefully. Only then does the audacity or boldness of rejecting a seemingly settled view amount to courage: for it reflects also significant preservation of values. Popper held that people must be able to be critical, and (piecemeal) to be bold in how they experiment, and in order to be able to replace some formerly received norm or idea. Popper's point about protecting freedom is that unless you do it, you actually do not secure security. For a society that lacks openness or critical rationality exposes itself to danger that might eventuate a drastic revolution which is utterly destructive of security.

Popper's anti-revolutionary dispositions deeply inform his philosophy of science, in ways that make what is "bold" appropriate and helpful precisely from its being courageous and only when it is courageous. Courage would not be possible in the revolutionary change, since courage requires significant protection of values. Revolutionaries overturn so much that their boldness can only be rash, not truly courageous. This is the implicit weight of a strong anti-revolutionary disposition in Popper.

\section{Conformity and Conservatism (Piecemeal) in Engineering Science}

Popper used the phrase "piecemeal engineering" to denote learning through trial and error; how we learn from our mistakes, recognise our errors and utilise them critically. The term "engineering" relates specifically to the application of science to solving problems. Engineering is the practical application of knowledge, and as such it is notable why Popper was endeared to it. However, it is doubtful if Popper's use of the term "engineering" carries the same meaning as the one actually implied by engineering science.

It is difficult to say whether any philosopher, Popper among them, had a proper understanding of the relationship between theoretical knowledge and engineering. To most philosophers, engineering is just an application of theoretical, tentatively accepted knowledge. Actual engineering science practice is in many domains based on a code. ${ }^{1}$ The code bears some relation to the sciences, such as physics. It is also strongly conditioned by practitioners' expediency. Actual engineering culture is careful by being conformist. Engineers themselves conform to codes, and expect one another's conformity to them. For example, there is a code for how you would calculate the strength of a bridge, say, by calculating the strength of bolts used

1 This is to acknowledge the contribution of Dr. Philip Catton to the development of this work. The knowledge of how actual engineering science practice works I got from my discussions with Dr. Catton, who transitioned from the field of philosophy to the field of civil engineering. 
for connecting one element of steel to another. This would involve simplified approximate assessments of six or seven different ways that failure is possible, checking which of these possible modes of failure is more likely under a given load, even taking into account a generous factor of safety. The engineering community will think that an engineer who has designed his bridge according to a code acted in an adequate way since engineering as a field demands careful code-conformity. And yet, the code itself may eventually be acknowledged to stand in need of revision or extension or other improvement. Science is not much present in the code, which is all about approximation for ease and quickness of calculation, but it becomes relevant at those occasions when the code is under review, and engineers are deciding how best to change it. This puts theory far from practice.

It is not clear if Popper had a good understanding of experimental science. This is because Popper focused too much on theory and the testing of hypotheses. Since Popper professed that we have no evidence for our theories, it will be difficult to ask Popper to explain why we should apply theoretical, tentatively accepted knowledge in the way that we do. Popper is theory-obsessed, just as most philosophers are theory-obsessed.

Although one could say that Popper may not have had a good understanding of how engineering culture works in practice, one may claim that Popper's anti-revolutionary stance on social and scientific reforms is indicative of his conformist view of the nature of engineering science. Popper seemed to have used the feature of engineering science regarding cross-checking possible modes of failure in order to endorse his idea of piecemeal engineering as a way of mitigating mistakes along the way. This is the fallibilism that Popper emphasised in his piecemeal engineering approach. This specifically accounts for the term "engineering," which Popper used to describe how the practical application of social and scientific reforms can be piecemeal. Although the method of engineering science may not be piecemeal, which was why Popper noted that there are "objectionable associations attached to the term engineering" (1957: 21), its heuristic nature involves making small changes in order to evaluate the steps taken. This is the approach that Popper also proposed in addressing social problems.

If engineering science is the creative application of scientific knowledge and skills to the natural, social and economic environment in order to design and create structures, machines, devices, systems, materials and processes, it is this underlying processes in engineering science that Popper wishes to adopt. So, when Popper used the word "engineering" to describe his piecemeal changes, he was interested in such engineering attitudes as that of completing a design by successive approximations, of making small changes at a time, and of retracting steps as well as exploring alternatives when failure occurs.

It is within this perspective that we can say that Popper used the term "engineering" to ascribe to the piecemeal engineer, technologist or scientist the responsibility to identify the problems at hand and begin to take a series of incremental steps to finding solutions to it. The engineer looks at an engineering design process, for instance, identifies a problem, say, how to build something (e.g. skyscraper, amusement park ride, bicycle, or music instrument). He/she gathers information and con- 
ducts research in order to understand the challenges to be addressed. Together with his/her team of engineers, they brainstorm many imaginative but potentially practical solutions. They select the most promising idea and embark upon a design that includes drawings, analytical decisions on the materials and construction, manufacturing and fabrication technologies to use. They create and test many prototypes, making improvements, analysing possible errors, learning from their mistakes until the product design is good enough to meet their professional needs.

In all of these steps, what the piecemeal engineer demonstrates is that the scientific process is a consciously designed one where engineers formulate the results of their designs in the form of hypotheses. They are able to identify the effects of the changes introduced because of the small adjustments and readjustments undertaken. The result of these small changes gives the engineer more information regarding which variable is to be changed and how much to change in it. This is the view that underwrites Popper's endorsement of piecemeal engineering, and neatly fits his account of science as conjectures and refutations. With this in mind, we can say that Popper's idea of piecemeal engineering is not only a method for solving social problems, and it is also applicable to the test of hypotheses in science.

P.D. Shaw (1971) and Gurol Irzik (1985, 2013) claimed that Popper's idea of piecemeal engineering expressed his approach to obtaining knowledge in natural science. Irzik interprets Popper as insisting upon the maxims (a) change as few variables as possible, and (b) make quantitatively small changes. Mindful of the scale and pace of change that is needed in a developing country such as his own native Turkey, Irzik criticises and rejects both maxim (a) and maxim (b).

Both Irzik and Shaw characterise Popper's piecemeal engineering as directly expressing the falsificationist idea that experimental science progresses through trial and error. How we learn from our mistakes, recognise our errors and utilise them critically (Popper 1957: 87), is crucial to Popper's proposed method of social transformation. The claim here is that in a typical liberal society it is relatively straightforward to detect social problems. Then, by trial and error, it is possible to assess policies aimed at solving the problems. In a society in which every rational person agrees where a problem lies, it is possible, by a process of experimentation analogous to that employed in natural science, to seek its solution. It is on the basis of the above claim that I shall examine the possibility of looking at Popper's piecemeal engineering as a scientific method.

\section{Piecemeal Engineering as a Scientific Approach}

Irzik (1985) critically discusses Popper's idea that the scientific approach of conjectures and refutations dovetails with his endorsement of piecemeal engineering. Popper contended, Irzik believes, that the method of changing the smallest possible number of variables in a piecemeal manner can aid the recognition of the consequences of these changes. That, then, according to Popper, represents an epistemic reason to be a piecemeal engineer doing "one-piece-at-a-time." Irzik's claim, however, is that while "one-piece-at-a-time" piecemeal engineering can facilitate 
error elimination, and can help us know the effects of the changes that we have made, it cannot get done very much within any reasonable amount of time. If, on the other hand, out of a need to accomplish considerable change in real time, we manipulate several variables at once, then, since the consequences will be a joint outcome of those multiple changes, it will often be extremely difficult to tell the role of each factor in producing these consequences. Moreover, some of these consequences will probably be undesired (Irzik 1985: 3).

It must be granted to Irzik that Popper is in a quandary here. Popper hoped that his epistemology would supply him with good reasons to be anti-revolutionary, but he took his argument to an unacceptable extreme. If one considers a country such as Irzik's native Turkey or the continent of Africa where I am from, where there are a great many socio-economic problems, upon which one would surely want work done many-pieces-at-once (Afisi 2012), then one does not have to be a revolutionary simply to want a good pace of improvement in real time. One may modify Popper's position and suggest that the researcher, by using the piecemeal, trial-and-error approach, may after all manipulate more than one variable at a time, and still may exert some control over other variables or measure change in other variables. Some conditions for ideal controlled experimentation would not apply, but the rationale would be that the pace of needed change cannot allow researchers what would be ideal conditions for them, conditions in which they manipulate one variable only, and control the rest of the variables within a controlled environment.

Whether the ideal experiment would involve a control group, where the subjects have been randomly assigned between the groups, and the researchers test only the effects that follow one change at a time, then that is in any case open to question. Society does not resemble the area of some well-defined branch of natural science. Society is highly dynamic, ramified and complicated. Ostensibly, researchers could know with greatest clarity the errors they have committed (when they think that a change that they can make will prove salutary and in fact it produces worse ill effects) only when one change occurs at a time. Society, however, won't allow for this anyway, even if the social engineer wants to follow such a one-changeat-a-time experimental method. Society will change at a significant pace and quite complexly, whether the social engineer orchestrates the change or not.

Irzik rightly points out that this approach of changing as few variables as possible is especially applicable in experimental designs using controlled variables and randomisation. It is also applicable where systematic tests can be carried out in classical experimental studies designed to isolate the separate ef fect of one variable on another (Irzik 1985: 3). Outside the special context of controlled scientific experimentation, it is dif ficult actually to achieve this piecemeal approach that Popper recommended. Yet Popper's piecemeal engineering approach could be combined with the techniques of regression analysis that social scientists use to carry out non-experimental research. The social scientists' aim is usually to infer from statistical data how an observed change in one variable is related to the separate changes in other variables operating simultaneously on it (Irzik 1985: 3). What this implies is that social scientists use regression analysis to gain an understanding of co-variation where they may not have 
full control of variables. Relatedly, then, piecemeal engineers could operate on more than one factor at once and potentially still learn from their mistakes. They would need to use statistics and regression analysis but by these means they could combine "many-pieces-at-once" piecemeal engineering with qualities of trial and error, learning from their mistakes in bits and pieces. The key issue, therefore, is limiting and controlling experimental variables so that causal factors can be identified. By identifying causes, the engineers can then attempt to have greater control of outcomes in later experiments. Irzik has helped us to see that this approach, by modifying Popper's view, can assume the form of "many-pieces-at-once." However, I insist that the manypieces-at-once piecemeal engineer, who is seeking to learn from mistakes made, is no holistic designer or revolutionary as Popper claimed.

Irzik has interpreted Popper's piecemeal engineering as directly expressing scientific method. We must acknowledge, however, that in every scientific inquiry hypotheses or theories are tested in bundles. A given hypothesis or theory can be tested only by combining it with auxiliary hypotheses. The French scientist and philosopher Pierre Duhem, in his The Aim and Structure of Physical Theory (1954 [1914]), has clarified and demonstrated the point in question. A standard illustration involves the case of celestial mechanics in which the way Uranus will be seen against the background of the fixed stars depends upon a complex of hypotheses. When scientists could not fit Uranus's orbit into their Newtonian models, they did not blame Newton's laws, rather they claimed that some further assumptions were required before they could predict Uranus's motion. It was fruitful for them to adjust their auxiliary assumptions, for as they were doing so, they discovered Neptune, a planet whose gravitational influence on Uranus had simply not been taken into account by the set of earlier auxiliary hypotheses. The auxiliary hypotheses at play in such inquiry need to be independently tested and testable. In testing these hypotheses scientists proceed through a piecemeal approach (and certainly not a wholesale approach) and this simply is the scientific method.

In spite of the endorsements of both Irzik and Shaw, not all commentators agree with this characterisation. For example, Michael Freeman (1975) denied that Popper's philosophy of science entails an incrementalist approach to scientific change, and so denied that the piecemeal reform that Popper favoured in social change is isomorphic with Popper's own conception of the process of natural science. Freeman's claim is that what Popper recommended in science is an act of revolutionary valour by way of falsification or error elimination and bold new conjecture, and this is contrary to the piecemeal change he favoured in social transformation.

Although Popper's piecemeal engineering uses conjectures and refutations, its orientation is social: piecemeal engineering seeks means to ameliorate an acknowledged social problem. As a method of solving social problems it concerns means/ ends, and not hypotheses alone. By contrast, Popper treats the testing of hypotheses in natural science as a purely intellectual concern. In Popper's view, any practical upshot would come later, as something fully separate from the advancement of theoretical knowledge. Though the methodologies of natural science on the one hand, and social engineering on the other, look quite alike, they are on this point 
completely different. Though Popper used the word engineering to describe a systematic elucidation of measures for proper social reform, the term "piecemeal engineering" is not synonymous with "the experimental process" in natural science. The practical intent of piecemeal engineering is an inseparable part of its meaning. Piecemeal engineering that Popper purported to define is the actual manipulation of society in order to reform it.

Moreover, we should be mindful of the fact that while there are well established laws of physics that the structural engineer or the physical scientist does take into account, there is much more controversy regarding the "laws of social dynamics" if indeed there are any. In fact, universal laws of science are not sufficient to understand the dynamics of the social environment. Although one would like hypotheses to be tested whenever possible, policies aimed at reforming have little to do with methodology in science.

Thus, piecemeal social engineering is for Popper done in the real world, especially in a particular, inevitably complex, social context. In this context, the social engineer hypothesises that doing $\mathrm{X}$ is a way (or is the best way) to bring about end E. For example, end $\mathrm{E}$ might be to improve public health, and the hypothesis might be that this is best brought about by doing $\mathrm{X}$, where, say, $\mathrm{X}=$ educating people about the effects of drinking untreated water. The point is that the engineer does not merely hypothesise, or adopt means for testing the hypothesis. On the contrary, the engineer also seeks to get something done, that is, to improve public health.

The engineer's means-end hypothesis is nevertheless open to test, and it might turn out true or false. The ways to test such a hypothesis are more various than either Popper, or with him Irzik, envisaged. One way to test the hypothesis is to use a control group that is randomly assigned and unaffected by X, with a contrasting experimental group subjected to X. This would be more like random clinical trials (RCT). Should the originally proffered hypothesis fail, one could begin, with the help of the data from such an RCT, a regression analysis towards shaping a new hypothesis. Since such RCT requires less stringent conditions than the conditions that would be demanded, according to either Popper or Irzik, by piecemeal engineering, I believe that the spirit of Popper's idea of piecemeal engineering would be satisfied, even if the one-piece-at-a-time requirement in his approach is inadequate. In my view, many-pieces-at-once social reform can count as piecemeal engineering.

\section{Conclusion}

I have argued that Popper's anti-revolutionary inclination deeply informed his piecemeal approach, in ways that make what is "bold" appropriate and helpful precisely because it is courageous and only when it is courageous. Courage, I have argued, would not be possible in outright revolutionary change, since courage requires preservation of values rather than their destruction. Revolutionaries overturn so much that their boldness can only be impetuous, not truly courageous. This is the implicit meaning of a strong anti-revolutionary outlook in Popper's 
philosophy. Popper downplays the extent to which a bold new conjecture in natural sciences can thoroughly "revolutionise" its field. Clearly, this position is in sharp contrast with Thomas Kuhn's concept of ,paradigm shift” which is the central idea of Kuhn's view of scientific revolution. The kind of change that Kuhn permits is a radical revolutionary fortitude that makes it mandatory to move to the stage of a paradigm shift when an "anomaly" in the given paradigm leads to a crisis situation (Kuhn 1962/1970: 66-76). Kuhn, therefore, believed that change must be a drastic one, once it becomes obvious that the paradigm no longer serves its purpose within normal science due to disconcerting anomalies. In such circumstances, a scientific revolution is inevitable; and the paradigm shift is ineluctable.

In opposition to this, Popper's conservative approach to change, his anti-revolutionary attitude in the piecemeal approach, sets him apart from many of other philosophers, including Kuhn. It may seem that Popper's idea of falsification requires that once a hypothesis becomes unsuccessful in the process of severe testing, it will necessarily lead to the rejection of a theory (Popper 1957: 86). However, one needs to understand that falsification in Popper connotes error elimination, and it is through criticisms and the piecemeal approach that one can cross-check a possible mode of failure along the same way in order to evaluate the major steps of changes, in a bid to mitigate mistakes along the way. This idea is the core of Popper's conservatism and its piecemeal approach. Against this, I have argued that Popper's conservative piecemeal approach to change requires modification in order to sufficiently address some concerns that a piecemeal approach might be insufficient. Accordingly, I suggested that an engineering approach of Popper involving "many-pieces-at-once" attitude is compatible with being able to learn from one's mistakes.

\section{Bibliography}

Afisi O.T., 2012, "Karl Popper on Piecemeal (or Many Pieces at Once) Social Engineering." A paper presented at the Australasia Postgraduate Philosophy Conference, held at the Department of Philosophy, University of Auckland, New Zealand 28-30 September 2012, www.Arts.auckland.ac.nz/uoa/karl-popper-on-piecemeal-social (accessed: 19.06.2019).

Catton P., 2010, "Matauranga Maori Refutes Popper," NZ Science Teacher 122, pp. 38-41.

Duhem P., 1954, The Aim and Structure of Physical Theory, transl. P.W. Wiener, Princeton [originally published as La Théorie Physique: Son Objet et sa Structure, Paris 1914].

Freeman M., 1975, "Sociology and Utopia: Some Reflections on the Social Philosophy of Karl Popper," British Journal of Sociology 26 [1], pp. 20-34.

Irzik G., 1985, "Popper's Piecemeal Engineering: What is Good for Science is not Always Good for Society," The British Journal for the Philosophy of Science, 36 [1], pp. 1-10. Irzik G., 2013, "Critical Rationalism," The Routledge Companion to the Philosophy of Science, S. Psillos, M. Curd (eds.), London-New York.

Kuhn T., 1962/1970, (second edition), The Structure of Scientific Revolution, Chicago. 
Popper K., 1957, The Poverty of Historicism, London.

Shaw P.D., "Popper, Historicism, and the Remaking of Society," Philosophy of the Social Sciences 1 (1971), pp. 299-308. 